\title{
CMV Infection: A Clinical Challenge in Biological Therapy? The Case of Asymptomatic Patients with Persistent Positive Immunoglobulin M Anti- CMV Treated with Secukinumab
}

This article was published in the following Dove Press journal:

Psoriasis: Targets and Therapy

\author{
Alessio Gambardella' \\ Gaetano Licata' \\ Giulia Calabrese' \\ Alina De Rosa' \\ Francesca Pagliuca ${ }^{2}$ \\ Roberto Alfano ${ }^{3}$ \\ Giuseppe Argenziano' \\ 'Dermatology Unit, Department of \\ Mental and Physical Health and \\ Preventive Medicine, University of \\ Campania Luigi Vanvitelli, Naples, Italy; \\ ${ }^{2}$ Division of Pathology, Department of \\ Mental and Physical Health and \\ Preventive Medicine, University of \\ Campania Luigi Vanvitelli, Naples, Italy; \\ ${ }^{3}$ Department of Anesthesiology, Surgery \\ and Emergency, University of Campania \\ Luigi Vanvitelli, Naples, Italy
}

\begin{abstract}
The use of biological therapy is now common practice in the treatment of immune-mediated inflammatory diseases (IMID). Currently, there are no guidelines related to the management of cytomegalovirus (CMV) infections or reactivation during therapy with biological agents. Furthermore, there is a lack of guidance on the management of asymptomatic patients with persistent positive immunoglobulin (Ig)M anti-CMV after an extended period and who have to undergo therapy with biological agents. We report the case of a patient in this situation for whom treatment with biological drugs for psoriasis was indicated. A good clinical response was obtained with secukinumab and maintained during 6 months of follow-up. No infectious disease or reactivation of CMV infection occurred. We suggest some possible guidelines for the management of such cases.
\end{abstract}

Keywords: immune-mediated inflammatory diseases, biological therapy, anti-TNF- $\alpha$ therapy, cytomegalovirus, psoriasis, case report

\section{Introduction}

Cytomegalovirus (CMV) infection is common in all human populations and is usually asymptomatic, leading to life-long persistence controlled by a strong $\mathrm{T}$ cellbased immunity. The use of biological agents is now a common practice in the treatment of immune-mediated inflammatory diseases (IMID), providing good control of the disease's progression and improving the patient's quality of life. Nevertheless, their use can cause an increased risk of new infectious events and/or reactivation of latent infectious diseases. ${ }^{1}$ Currently, there are no guidelines related to the management of CMV infections or reactivation during therapy with biological agents. In the literature, limited data are available on the use of anti-tumor necrosis factor (TNF)- $\alpha$ therapy in this condition. Furthermore, screening for CMV infections is not recommended before starting biological therapy, ${ }^{2}$ and there is a lack of guidelines on the management of particular cases, such as asymptomatic patients with persistent positive immunoglobulin (Ig)Manti-CMV after an extended period and who have to undergo therapy with biological agents. No cases of patients with this serological state are described in the literature. Here we report the case of a patient with this condition treatment who needed to start treatment with biological drugs for psoriasis and suggest some possible guidelines for the management of such cases.
Correspondence: Gaetano Licata Department of Dermatology, University of Campania "Luigi Vanvitelli", Via Sergio Pansini, 5, Napoli 80I3I, Italy

Tel +390815666828

Fax +390815468759

Email gaetano.licata89@gmail.com 


\section{Case Report}

A 61-year-old man with psoriasis since the age of 32 years and a past medical history of family hypercholesterolemia was hospitalized in May 2018 with fever $\left(40^{\circ} \mathrm{C}\right)$, pharyngitis, and general malaise. On physical examination, enlargement of the spleen, liver, and lymph nodes was observed. Biochemical analysis revealed a white cell count of $12,000 / \mathrm{mm}^{3}$, elevations of hepatic transaminases (aspartate aminotransferase [AST] $120 \mathrm{IU} / \mathrm{L}$, alanine aminotransferase $[\mathrm{ALT}] 111 \mathrm{IU} / \mathrm{L}$ ) and the presence of atypical lymphocytosis. Human immunodeficiency virus (HIV), hepatitis B virus (HBV), hepatitis C virus (HCV), EpsteinBarr virus (EBV), CMV, and anti-neutrophil cytoplasmic autoantibodies (ANCA) serology screenings were performed in order to exclude any underlying cause, with normal results, except for IgM anti-CMV $>30 \mathrm{UA} / \mathrm{mL}$. A diagnosis of acute CMV infection was made.

The patient was observed over time, and four weeks later his clinical manifestations disappeared and his blood tests returned to normal. One year later, the patient was referred to our department. On dermatological examination, we observed many erythematous-squamous plaques on his bilateral lower limbs, lumbar area, back, and trunk. These clinical features suggested the diagnosis of moderate-to-severe psoriasis, with a Psoriasis Area and Severity Index (PASI) score of 16, body surface area (BSA) score of 12 and a Dermatology Life Quality Index (DLQI) score of 18 (Figure 1A and B). The patient had previously been treated with topical steroid therapy, ultraviolet (UV)B narrow-band phototherapy for about a year before discontinuing due to lack of efficacy, and treatment with $20 \mathrm{mg}$ of methotrexate per week for about three years before stopping because of poor response. Taking this into account, the patient was eligible for therapy with biological agents, and considering the positive anamnesis for CMV infection, a careful screening was carried out; the patient did not have any clinical symptoms or sign of viral infection syndrome at the time of screening, and results were all within the normal range, except for IgM anti$\mathrm{CMV}>28 \mathrm{UA} / \mathrm{mL}$ and positive IgG anti-CMV. Considering these results, the patient had a consultation with the infectious disease specialist to determine if it was a new infection or a reactivation. Subsequently, IgG antiCMV avidity and CMV-polymerase chain reaction (PCR) were ordered, with negative results. Having established that it was a previous infection, the infectious disease specialist determined that the patient could undertake therapy with a biological drug while monitoring $\operatorname{IgG}$ antiCMV avidity and CMV-PCR every three months. In June 2019, after informed consent was obtained, we started treatment with secukinumab, an anti-interleukin -17A monoclonal antibody approved for the treatment of moderate-to-severe plaque psoriasis, at a dose of $300 \mathrm{mg}$ by subcutaneous injection with initial dosing at weeks 0,1 , 2,3 and 4, followed by monthly maintenance dosing. A good clinical response was obtained after 16 weeks of secukinumab (PASI 2, BSA 0, DLQI 0) (Figure 1C and D) and was maintained during a 6-month follow-up. No infectious disease or reactivation of CMV infection or other adverse events were observed during the follow-up period. IgG anti-CMV avidity and CMV-PCR were negative after three, six, nine, and twelve months from starting therapy.

\section{Discussion}

Biological therapies have revolutionized the treatment of various immune diseases, but in any patient receiving these drugs it is important to be conscious of infections because there is a risk of a long and aggressive course. CMV and EBV reactivations have been reported in patients receiving biologics, especially anti-TNF- $\alpha$ agents. ${ }^{3}$ In a brief review of the literature, Mencarini et al identified 14 cases of CMV infections during biological therapy with anti-TNF- $\alpha$ in IMID patients. ${ }^{4}$ It was not clearly described whether the infections were primary or reactivations. Nevertheless, temporary discontinuation of the biological drug led to the improvement of the patients' condition in almost all cases. ${ }^{4}$ However, there is a lack of guidelines in the case of asymptomatic patients who have had a CMV infection with positive IgM anti-CMV and IgG anti-CMV and who need to undergo therapy with biological agents. Indeed, the presence of IgM anti-CMV in the bloodstream is not always characteristic of the acute/recent phase of the infection. Conversely, their presence can often be detected for a long period after infection. In addition, IgM antiCMV may also be detectable in secondary infections (reinfections or reactivations). ${ }^{5,6}$

In such cases of difficult interpretation in determining whether the infection is an acute/recent infection or a previous infection, we propose that the following tests are carried out: IgG anti-CMV avidity and CMV-PCR. The IgG anti-CMV avidity test can detect an acute infection with $92-100 \%$ sensitivity and $82-100 \%$ specificity. ${ }^{7}$ A high level of IgG anti-CMV avidity is highly suggestive of a prior infection and excludes primary infection within the preceding 3 months, while, on the contrary, a low level 

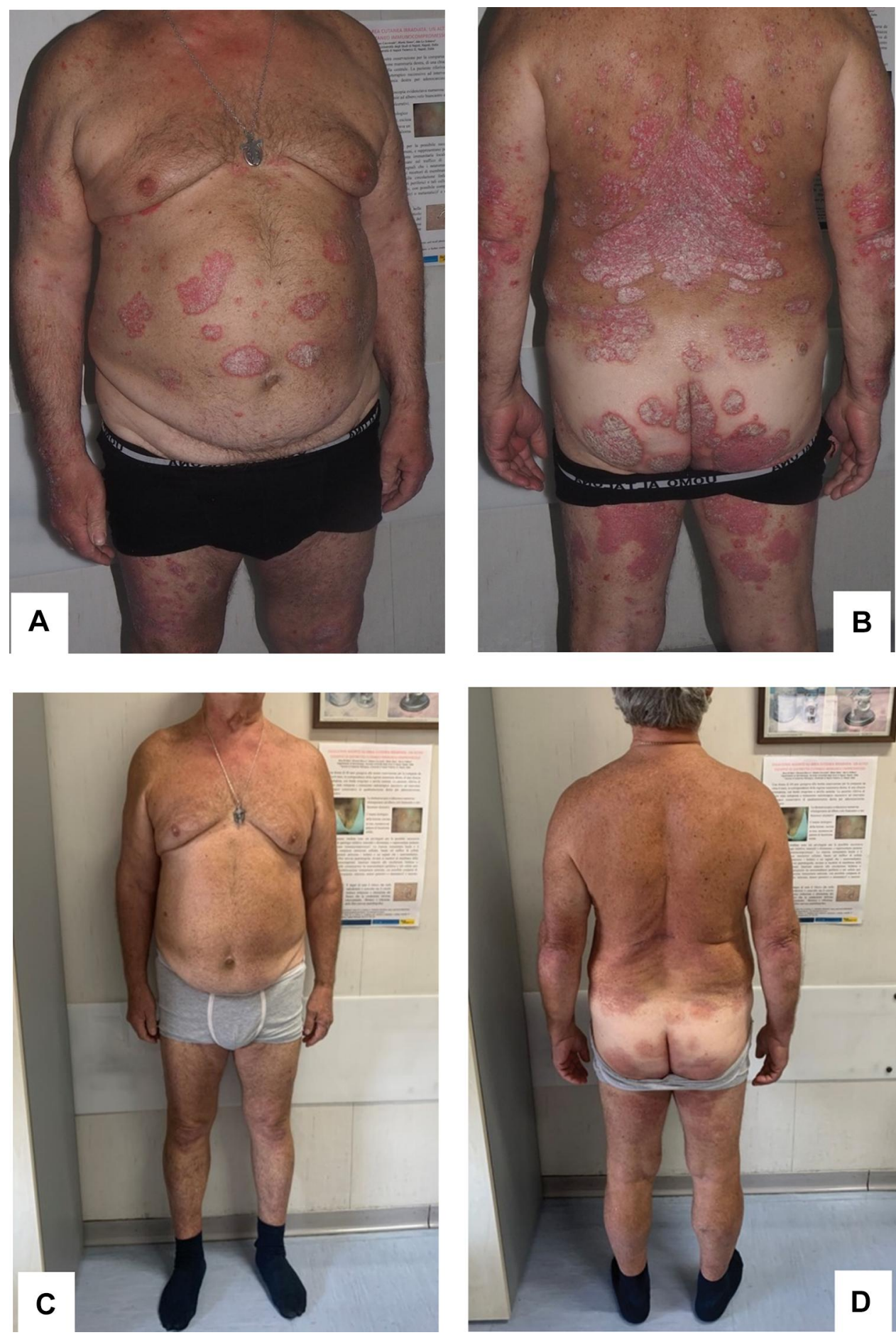

Figure I (A and B) Moderate-to-severe psoriasis, with a Psoriasis Area and Severity Index (PASI) score of I6, body surface area (BSA) score of I2 and Dermatology Life Quality Index (DLQI) score of 18 prior to treatment with biological therapy. (C and D) Complete clinical response after 16 weeks of secukinumab.

of IgG anti-CMV avidity is an accurate indicator of primary infection within the preceding 3 to 4 months and strongly suggests infection in the acute phase. Another valuable test is real-time PCR for the quantitative determination of CMV DNA; this exam has a sensitivity of $100 \%$ and specificity $\geq 99 \%{ }^{8}$ Thanks to these two tests, it is possible to manage these complex situations. In the case of our patient, although IgM anti-CMV were still present, once it was established that the patient had a previous infection, we started therapy with biological drugs. 
In the literature, there is limited data on the safety of biological agents in patients with CMV infections. In our case, the biological drug chosen was secukinumab, due to its high safety profile. Through the SCULPTURE study (Study Comparing secukinumab Use in Long-term Psoriasis maintenance therapy: fixed regimens vs reTreatment Upon start of RElapse), it has been shown that the anti-17A monoclonal antibody has a highly favorable safety profile, with the favorable tolerability at one year maintained through five years of treatment. ${ }^{9}$ The most common adverse events in the overall study population were nasopharyngitis, back pain, and headache, and there were no cases of viral infections or reactivation of previous viral infections. ${ }^{9}$ Furthermore, Chiu et al performed a study of the impact of long-term secukinumab treatment on EBV and CMV loads in patients with psoriasis. No significant change from baseline plasma CMV or EBV loads was found after treatment with secukinumab. ${ }^{10}$ These findings confirm the clinical safety of IL-17A antagonists. In conclusion, among the biological drugs available for psoriasis, the IL-17A antagonists have proven to be the safest in terms of viral reactivation.

To guide treatment decisions in the case of asymptomatic patients with positive IgM anti-CMV, we recommend carrying out IgG anti-CMV avidity and CMV-PCR tests to clarify whether the infection is acute or if it is a past infection. Further studies with larger numbers of patients are needed to evaluate the effectiveness of this proposed guideline.

\section{Ethics Statement}

No institutional approval was required to publish the case details.

\section{Patient Consent for Publication Statement}

The patient provided written informed consent to the publication of his case details and images.

\section{Acknowledgments}

We thank Ray Hill, an independent medical writer, who provided English-language editing and journal styling prior to submission on behalf of Health Publishing \& Services Srl. This unconditional support was funded by Novartis Farma SpA. This research did not receive any specific grant from funding agencies in the public, commercial, or not-for-profit sectors.

\section{Disclosure}

The authors declare no conflicts of interest in this work.

\section{References}

1. Petersen B, Lorentzen H. Cytomegalovirus complicating biological immunosuppressive therapy in two patients with psoriasis receiving treatment with etanercept or efalizumab. Acta Derm Venereol. 2008;88(5):523-524. doi:10.2340/00015555-0491

2. Miquel FJ, Colomina J, Marii JI, Ortega C. Cytomegalovirus infection in a patient treated with efalizumab for psoriasis. Arch Dermatol. 2009;145(8):961-962. doi:10.1001/archdermatol.2009.154

3. Caudron A, Lok C, Clabaut A, Desblache J, Heyman B, Viseux V. Cytomegalovirus colitis in a patient treated with efalizumab. Dermatology. 2009;218(1):84-85. doi:10.1159/000174038

4. Mencarini J, Spinicci M, Bartalesi F. Risk of cytomegalovirus reactivation in patients with immune-mediated inflammatory diseases undergoing biologic treatment: a real matter? Reumatismo. 2016;68 (3):144-147. doi:10.4081/reumatismo.2016.897

5. Krmpotić A, Podlech J, Reddehase MJ, Britt WJ, Jonjić S. Role of antibodies in confining cytomegalovirus after reactivation from latency: three decades' resume. Med Microbiol Immunol. 2019;208 (3-4):415-429. doi:10.1007/s00430-019-00600-1

6. Sissons JG, Carmichael AJ. Clinical aspects and management of cytomegalovirus infection. $J$ Infect. 2002;44(2):78-83. doi:10.1053/ jinf.2001.0949

7. Vauloup-Fellous C, Lazzarotto T, Revello MG, Grangeot-Keros L. Clinical evaluation of the Roche Elecsys CMV IgG avidity assay. Eur J Clin Microbiol Infect Dis. 2014;33(8):1365-1369. doi:10.1007/ s10096-014-2080-4

8. Ross SA, Novak Z, Pati S, Boppana SB. Overview of the diagnosis of cytomegalovirus infection. Infect Disord Drug Targets. 2011;11 (5):466-474. doi:10.2174/187152611797636703

9. Bissonnette R, Luger T, Thaci D, et al. Secukinumab demonstrates high sustained efficacy and a favourable safety profile in patients with moderate-to-severe psoriasis through 5 years of treatment (SCULPTURE Extension Study). J Eur Acad Dermatol Venereol. 2018;32(9):1507-1514. doi:10.1111/jdv.14878

10. Chiu HY, Chan CC, Tsai TF. The impact of long-term secukinumab treatment on Epstein-Barr virus and cytomegalovirus loads in patients with psoriasis. Int J Dermatol. 2016;55(11):e600-e602. doi:10.1111/ ijd.13346
Psoriasis: Targets and Therapy

\section{Publish your work in this journal}

Psoriasis: Targets and Therapy is international, peer-reviewed, open access journal focusing on psoriasis, nail psoriasis, psoriatic arthritis and related conditions, identification of therapeutic targets and the optimal use of integrated treatment interventions to achieve improved outcomes and quality of life. Visit http://www.dovepress. com/testimonials.php to read real quotes from published authors. 